\title{
A time-domain substructure synthesis for finite rotations of flexible mechanical systems
}

\author{
Shulin Chen ${ }^{1}$, Li Liu ${ }^{2}$ and Zhaoyue Chen ${ }^{3}$ \\ ${ }^{1,2,3}$ School of Aerospace Engineering, Beijing Institute of Technology, Zhongguancun South Street 5, 100081 Beijing, China
}

\begin{abstract}
This paper presents a floating IBS method for dynamic prediction of structures with finite rotations. The conventional IBS (impulse-based substructuring) method is efficient and accurate in structural transient impact analysis. When facing about finite rotations, the conventional IBS method will generate unreasonable results due to the lack of consideration of geometric nonlinearity. In this paper, the idea of floating frame of reference is introduced. The motions of structures are divided into the moving of floating frame of reference and local vibrations which are described using the idea of IBS method. Two numerical examples validate that the proposed method is available for unconstrained single-body system (free-free rotating beam) and constrained multibody system (a slider-crank system). Meanwhile, to some extent the local elastic vibration degrees of freedom can be reduced by employing interpolation matrixes
\end{abstract}

\section{Introduction}

The increase of spacecraft's complexity has rose computational costs of dynamics simulation and design optimization enormously. In order to cut down computing time and improve computational efficiency, dynamic substructuring (DS) techniques emerged.

The DS idea was first accomplished by Hurty in 1960 [1], and now DS have been widely employed in many industrial fields. In general, DS can be classified as: component mode synthesis (CMS), frequency-based substructuring (FBS) methods [2], and impulse-based substructuring (IBS) methods [3]. For more about DS techniques, refer to [4]. In 2010, Rixen first proposed the form of IBS and successfully achieved the implementation of the IBS technique using either numerical IRFs or measured IRFs [5]. After this some further development has been studied $[6,7]$. IBS has been proven to be effective in dealing with transient impact problems [3], such as the soft landing process of spacecraft. Dong first achieved the implementation of the IBS technique in dynamic analysis of lunar lander and made some improvement which makes it possible to consider rigid and linear or non-linear elastic connections between substructures using IRFs $[8,9]$.

However, for the dynamic prediction of complex spacecraft, there may be geometric nonlinearity, such as the rotation of the solar wing about its hinges and the finite rotation of the lunar lander when it lands on the inclined plane. When structures make finite rotation, the conventional IBS method will lead to an unreasonable result. In order to overcome this shortcoming, we propose a floating IBS, which is based on the thought of floating frame of reference. When a body undergoes finite rotation, the actual motion of the system can be divided into the rigid motion of a floating body frame and the linear local elastic vibration with respect to the floating body reference [10]. Then the local elastic vibration can be expressed using the idea of IBS method. To reduce elastic degrees and improve the computational efficiency, an interpolation matrix is introduced. Two numerical examples are given to illustrate the proposed method.

\section{Flexible multibody system using floating frame of reference}

For simplicity, we take a planar analysis to explain how floating frame of reference works. First we discrete the flexible body by finite element method. Then we define three references, as shown in Fig. 1: the inertial reference (OXY), the floating reference (OfXfYf) and the element reference.

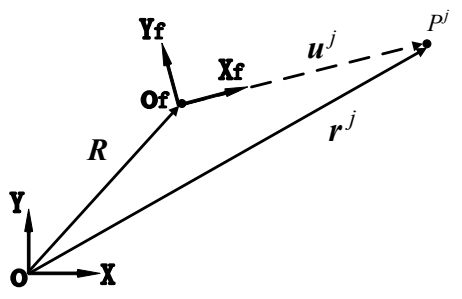

Figure. 1 The illustration of floating frame of reference

The inertial reference is fixed to ground to describe the whole system. The floating reference moves with the flexible body, under which the relative motion of the element node is described. The element reference is 
defined in finite element discrete process and has a fixed angle with the floating reference [1].

For node $j$, its displacement can be described in the inertial reference

$$
\boldsymbol{r}^{j}=\left[\begin{array}{ll}
x^{j} & y^{j}
\end{array}\right]^{\mathbf{T}}
$$

and its displacement under the floating reference is

$$
\boldsymbol{u}^{j}=\left[\begin{array}{ll}
\bar{x}^{j} & \bar{y}^{j}
\end{array}\right]^{\mathbf{T}}
$$

Meanwhile, the floating reference's rigid displacement is described as

$$
\boldsymbol{R}=\left[\begin{array}{ll}
x & y
\end{array}\right]^{\mathbf{T}}
$$

For a planar analysis, we have a transform matrix between the floating reference and the inertial reference

$$
\boldsymbol{A}=\left[\begin{array}{cc}
\cos \theta & -\sin \theta \\
\sin \theta & \cos \theta
\end{array}\right]
$$

where $\theta$ is rotational angle between floating reference and inertial reference. Then, there is a relationship

$$
\boldsymbol{r}^{j}=\boldsymbol{R}+\boldsymbol{A} \boldsymbol{u}^{j}
$$

As described in finite element frame, $\boldsymbol{u}^{j}$ can be expressed as

$$
\boldsymbol{u}^{j}=\boldsymbol{D}^{j} \boldsymbol{S}^{j} \boldsymbol{B}_{1}^{j} \boldsymbol{q}
$$

where $\boldsymbol{q}$ is element displacement, $\boldsymbol{S}^{j}$ is element shape function, $\boldsymbol{B}_{1}^{j}$ is a Boolean matrix used to project all generalized displacement to an element, $\boldsymbol{D}^{j}$ is a transform matrix between the floating reference and the element reference through a rotational angle $\beta^{j}$.

$$
\boldsymbol{D}^{j}=\left[\begin{array}{cc}
\cos \beta^{j} & -\sin \beta^{j} \\
\sin \beta^{j} & \cos \beta^{j}
\end{array}\right]
$$

Now we divide element displacement $\boldsymbol{q}$ into initial displacement $\boldsymbol{q}_{0}$ and elastic displacement $\boldsymbol{q}_{f}$. To avoid rigid motion, a constrained condition is introduced and there is $\boldsymbol{q}=\boldsymbol{q}_{0}+\boldsymbol{B}_{2} \boldsymbol{q}_{\boldsymbol{f}}, \boldsymbol{B}_{2}$ is a Boolean matrix used to

$$
\left[\begin{array}{cc}
M_{R R} & A_{\theta} I_{0}+A_{\theta} S_{1} B_{2} q_{f} \\
& I_{02}+2 I_{03} B_{2} q_{f}+q_{f}^{\mathrm{T}} B_{2}^{\mathrm{T}} S_{2} B_{2} q_{f} \\
\text { symmetry } &
\end{array}\right.
$$

$$
\begin{aligned}
& =\left[\begin{array}{r}
\dot{\theta}^{2} A\left(I_{0}+S_{1} B_{2} q_{f}\right)-2 \dot{\theta} A_{\theta} S_{1} B_{2} \dot{q}_{f} \\
-2 \dot{\theta} \dot{q}_{f}^{\mathrm{T}} B_{2}^{\mathrm{T}} S_{2} B_{2} q_{f}-2 \dot{\theta} \dot{q}_{f}^{\mathrm{T}} B_{2}^{\mathrm{T}} I_{03}^{\mathrm{T}} \\
\dot{\theta}^{2}\left(I_{03} B_{2} I_{f}+I_{f}^{\mathrm{T}} B_{2}^{\mathrm{T}} S_{2} B_{2} q_{f}\right)+2 \dot{\theta} I_{f}^{\mathrm{T}} B_{2}^{\mathrm{T}} S_{3}
\end{array}\right. \\
& \text { tation of ibs algorithm to } \\
& \text { tibody system }
\end{aligned}
$$
flexible multibody system

The IBS methods implement the convolution (i.e. the Duhamel's integral) of the IRFs matrix and the applied force vector to represent the dynamic responses of a substructure and use interface compatibility and equilibrium to assemble adjacent substructures. It can be described as constrain rigid motion. Defining a constant matrix $\boldsymbol{N}^{j}=\boldsymbol{D}^{j} \boldsymbol{S}^{j} \boldsymbol{B}_{1}^{j}$, we have

$$
\boldsymbol{r}^{j}=\boldsymbol{R}+\boldsymbol{A} \boldsymbol{u}^{j}=\boldsymbol{R}+\boldsymbol{A} \boldsymbol{N}^{j}\left(\boldsymbol{q}_{0}+\boldsymbol{B}_{2} \boldsymbol{q}_{\boldsymbol{f}}\right)
$$

Taking a derivation of $\boldsymbol{r}^{j}$ by time, we get

$$
\dot{\boldsymbol{r}}^{j}=\left[\begin{array}{lll}
\boldsymbol{I} & A_{\theta} \boldsymbol{N}^{j}\left(\boldsymbol{q}_{0}+\boldsymbol{B}_{2} \boldsymbol{q}_{f}\right) & A \boldsymbol{N}^{j} B_{2}
\end{array}\right] \dot{\boldsymbol{Q}}
$$

where $\dot{\boldsymbol{Q}}$ is the generalized velocity and

$$
\dot{\boldsymbol{A}}=\boldsymbol{A}_{\theta} \dot{\theta}, \quad \boldsymbol{A}_{\theta}=\left[\begin{array}{cc}
-\sin \theta & -\cos \theta \\
\cos \theta & -\sin \theta
\end{array}\right], \quad \dot{\boldsymbol{Q}}=\left[\begin{array}{lll}
\dot{\boldsymbol{R}}^{\mathrm{T}} & \dot{\theta} & \dot{\boldsymbol{q}}_{f}^{\mathrm{T}}
\end{array}\right]^{\mathbf{T}} \text {. }
$$

Then the kinetic energy of dynamic system can be expressed as

$$
\boldsymbol{T}^{j}=1 / 2 \int \rho^{j} \dot{\boldsymbol{r}}^{j \mathbf{T}} \dot{\boldsymbol{r}}^{j} d V^{j}
$$

where $\rho$ is the material density, $V$ is the element volume.

Consider the Lagrange equation

$$
\frac{d}{d t}\left(\frac{\partial \boldsymbol{T}^{j}}{\partial \dot{\boldsymbol{Q}}}\right)^{\mathbf{T}}-\left(\frac{\partial \boldsymbol{T}^{j}}{\partial \boldsymbol{Q}}\right)^{\mathbf{T}}+\boldsymbol{C}_{Q}^{\mathbf{T}} \lambda_{c}=\boldsymbol{F}_{g}^{j}
$$

where $\boldsymbol{F}_{g}^{j}$ is generalized external force, $\boldsymbol{C}_{\boldsymbol{Q}}$ is the Jacobian matrix of system constraint function, $\lambda_{c}$ is the Lagrange multiplier for system constraint.

Substituting Eq.(9) and Eq.(10) into Eq.(11), yields

Eq.(12), which is the dynamic function under the floating frame of reference. In Eq.(12), ne is the number of element, $\boldsymbol{F}_{\text {out }}$ is external force vector. And we define the constants:

$$
\boldsymbol{S}_{1} \equiv \sum_{j}^{n e} \int_{V} \rho \boldsymbol{N}^{j} \mathrm{~d} V, \quad \boldsymbol{S}_{2} \equiv \sum_{j}^{n e} \int_{V} \rho \boldsymbol{N}^{j \mathbf{T}} \boldsymbol{N}^{j} \mathrm{~d} V,
$$$$
\boldsymbol{S}_{3} \equiv \sum_{j}^{n e} \int_{V} \rho \boldsymbol{N}^{j \mathrm{~T}} \tilde{\boldsymbol{I}} \boldsymbol{N}^{j} \mathrm{~d} V, \quad \boldsymbol{I}_{0} \equiv \sum_{j}^{n e} \int_{V} \rho \boldsymbol{N}^{j} \boldsymbol{q}_{0} \mathrm{~d} V,
$$$$
\boldsymbol{I}_{02} \equiv \sum_{j}^{n e} \int_{V} \rho \boldsymbol{q}_{0}^{T} \boldsymbol{N}^{j \boldsymbol{T}} \boldsymbol{N}^{j} \boldsymbol{q}_{0} \mathrm{~d} V, \quad \boldsymbol{I}_{03} \equiv \sum_{j}^{n e} \int_{V} \rho \boldsymbol{q}_{0}^{\mathbf{T}} \boldsymbol{N}^{j \mathbf{T}} \boldsymbol{N}^{j} \mathrm{~d} V,
$$$$
\boldsymbol{A}_{\theta}^{\boldsymbol{T}} \boldsymbol{A}=\left[\begin{array}{cc}
0 & 1 \\
-1 & 0
\end{array}\right] \equiv \tilde{\boldsymbol{I}} \text {. }
$$

$\left.\begin{array}{c}\boldsymbol{A} \boldsymbol{S}_{1} \boldsymbol{B}_{2} \\ \boldsymbol{I}_{04}+\boldsymbol{q}_{f}^{\mathrm{T}} \boldsymbol{B}_{2}^{\mathrm{T}} \boldsymbol{S}_{3} \boldsymbol{B}_{2} \\ \boldsymbol{B}_{2}^{T} \boldsymbol{S}_{2} \boldsymbol{B}_{2}\end{array}\right]\left[\begin{array}{c}\ddot{\boldsymbol{R}} \\ \ddot{\theta} \\ \ddot{\boldsymbol{q}}_{f}\end{array}\right]+\left[\begin{array}{ccc}0 & 0 & 0 \\ 0 & 0 & 0 \\ 0 & 0 & \boldsymbol{K}_{f f}\end{array}\right]\left[\begin{array}{c}\boldsymbol{R} \\ \theta \\ \boldsymbol{q}_{f}\end{array}\right]+\boldsymbol{C}_{Q}^{\mathrm{T}} \boldsymbol{\lambda}_{\boldsymbol{c}}$

$$
\left\{\begin{array}{l}
\boldsymbol{u}^{(s)}(t)=\int_{0}^{t} \boldsymbol{H}^{(s)}(t-\tau)\left(\boldsymbol{f}^{(s)}(\tau)+\boldsymbol{g}^{(s)}(\tau)\right) \mathrm{d} \tau \\
\boldsymbol{B} \boldsymbol{u}=\sum_{s=1}^{N_{s}} \boldsymbol{B}^{(s)} \boldsymbol{u}^{(s)}=\boldsymbol{0}
\end{array}\right.
$$

where superscript $s$ denotes the serial number of substruc tures, $\boldsymbol{u}^{(s)}$ is the displacement vector of substructure $s$, $\boldsymbol{H}(t)$ is the impulse response functions (IRFs) matrix, $\boldsymbol{f}(t)$ is the external applied force vector, $\boldsymbol{g}(t)$ is the interf ace force vector, $N_{s}$ is the number of substructures and $\boldsymbol{B}$ is a signed Boolean matrix identifying the degrees of freed oms (DOFs) on the interfaces. More details refer to [8]. 
As shown in Eq.(12), $\boldsymbol{q}_{f}$ is the elastic displacement. Under the floating frame of reference, solving $\boldsymbol{q}_{f}$ is still a linear elasticity problem. Following the idea of IBS, we use the Duhamel integral

$$
\boldsymbol{q}_{f}=\int_{0}^{t} \boldsymbol{H}(t-\tau) \lambda(t) \mathrm{d} \tau
$$

where $\lambda(t)$ is the coupling factor of floating reference's rigid motion and local elastic vibration.

First we consider one flexible body system. Then we ignore the system constraint and divide Eq.(12) by floating reference's rigid motion and local elastic vibration

$$
\left[\begin{array}{ll}
\boldsymbol{M}_{t t} & \boldsymbol{M}_{t f} \\
\boldsymbol{M}_{t f}^{T} & \boldsymbol{M}_{f f}
\end{array}\right]\left[\begin{array}{l}
\ddot{\boldsymbol{q}}_{t} \\
\ddot{\boldsymbol{q}}_{f}
\end{array}\right]+\left[\begin{array}{cc}
0 & 0 \\
0 & \boldsymbol{K}_{f f}
\end{array}\right]\left[\begin{array}{l}
\boldsymbol{q}_{t} \\
\boldsymbol{q}_{f}
\end{array}\right]=\boldsymbol{Q}_{v}
$$

where $\boldsymbol{q}_{t}=\left[\begin{array}{ll}\boldsymbol{R}^{T} & \theta\end{array}\right]^{T}$ and $\boldsymbol{Q}_{v}$ stands for right part of Eq.(12).

Write Eq.(15) in a difference scheme

$$
\boldsymbol{M}_{n+1}\left[\begin{array}{c}
\ddot{\boldsymbol{q}}_{t} \\
\ddot{\boldsymbol{q}}_{f}
\end{array}\right]_{n+1}+\boldsymbol{K}_{n+1}\left[\begin{array}{c}
\boldsymbol{q}_{t} \\
\boldsymbol{q}_{f}
\end{array}\right]_{n+1}=\boldsymbol{Q}_{v n+1}
$$

where

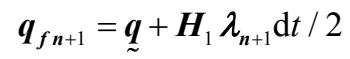

subscript $n$ means that the variable is at $n \mathrm{~d} t$ time, $\mathrm{d} t$ is the time step and

$$
\underset{\sim}{\boldsymbol{q}}=\sum_{i=0}^{n-1} \boldsymbol{H}_{n-i}\left(\lambda_{i}+\lambda_{i+1}\right) \mathrm{d} t / 2+\boldsymbol{H}_{1} \lambda_{n} \mathrm{~d} t / 2
$$

Introduce the Newmark integration strategy

$$
\left\{\begin{array}{l}
\ddot{\boldsymbol{q}}_{n+1}=\frac{1}{\beta \mathrm{d} t^{2}} \boldsymbol{q}_{n+1}+\ddot{\boldsymbol{Q}}_{n}^{\mathrm{int}} \\
\dot{\boldsymbol{q}}_{n+1}=\frac{\gamma}{\beta \mathrm{d} t} \boldsymbol{q}_{n+1}+\dot{\boldsymbol{Q}}_{n}^{\text {int }}
\end{array}\right.
$$

where

$$
\begin{aligned}
& \ddot{\boldsymbol{Q}}_{n}^{\mathrm{int}}=-\frac{1}{\beta \mathrm{d} t^{2}} \boldsymbol{q}_{n}-\frac{1}{\beta \mathrm{d} t} \dot{\boldsymbol{q}}_{n}-\left(\frac{1}{2 \beta}-1\right) \ddot{\boldsymbol{q}}_{n}, \\
& \dot{\boldsymbol{Q}}_{n}^{\mathrm{int}}=-\boldsymbol{q}_{n} \frac{\gamma}{\beta \mathrm{d} t}+\left(1-\frac{\gamma}{\beta}\right) \dot{\boldsymbol{q}}_{n}+\left(1-\frac{\gamma}{2 \beta}\right) \ddot{\boldsymbol{q}}_{n} \mathrm{~d} t .
\end{aligned}
$$

Substituting Eq.(19) and Eq.(17) into Eq.(16), yields

$$
\begin{aligned}
& {\left[\begin{array}{cc}
\boldsymbol{M}_{t t} & \boldsymbol{M}_{t f} \boldsymbol{H}_{1} \mathrm{~d} t / 2 \\
\boldsymbol{M}_{t f}^{T} & \left(\boldsymbol{M}_{f f}+\beta \mathrm{d} t^{2} \boldsymbol{K}_{f f}\right) \boldsymbol{H}_{1} \mathrm{~d} t / 2
\end{array}\right]\left[\begin{array}{l}
\boldsymbol{q}_{t} \\
\lambda
\end{array}\right]_{n+1}} \\
& =\left(\boldsymbol{Q}_{v n+1}-\boldsymbol{M}_{n+1} \ddot{\boldsymbol{Q}}_{n}^{\text {int }}\right) \beta \mathrm{d} t^{2}-\left[\begin{array}{c}
\boldsymbol{M}_{t f} \underset{\sim}{\boldsymbol{q}} \\
\left(\boldsymbol{M}_{f f}+\beta \mathrm{d} t^{2} \boldsymbol{K}_{f f}\right) \boldsymbol{q}
\end{array}\right]
\end{aligned}
$$

Solving Eq.(20) by Newton-Raphson method, we get $\left[\begin{array}{ll}q_{t} & \lambda\end{array}\right]_{n+1}^{\mathrm{T}}$. Then $\boldsymbol{q}_{f n+1}$ is obtained by substituting $\lambda_{n+1}$ into Eq.(17).

In order to reduce the elastic degrees, interpolation matrix $\mathbf{T}$ can be introduced

$$
\boldsymbol{q}_{f}=\mathbf{T} \overline{\boldsymbol{q}}_{f}
$$

Eq.(14) now changes into

$$
\boldsymbol{q}_{f}=\mathbf{T} \int_{0}^{t} \overline{\boldsymbol{H}}(t-\tau) \bar{\lambda}(t) \mathrm{d} \tau
$$

Because of the introduction of $\mathbf{T}$, the dimension of the impulse response function matrix and coupling factor reduces. In this paper we use the Lagrange interpolation function as the interpolation matrix, nevertheless other interpolation function can be used.

For multibody system, the system constraint between adjacent bodies should be considered.

$$
\boldsymbol{C}(\boldsymbol{Q}, t)=\mathbf{0}
$$

where $\boldsymbol{C}$ is the linearly independent constraint equations.

Derivation of Eq.(23) by time once and twice separately yields

$$
C_{Q} \dot{Q}+C_{t}=\mathbf{0}
$$

and

$$
C_{Q} \ddot{Q}+C_{t t}+2 C_{Q t} \dot{Q}+\left(C_{Q} \dot{Q}\right)_{Q} \dot{Q}=0
$$

where the subscript $\boldsymbol{Q}$ and $t$ separately stand for the partial derivation by generalized displacement vector $\boldsymbol{Q}$ and time $t$, thus $\boldsymbol{C}_{\boldsymbol{Q}}$ is the Jacobian matrix of system constraint function.

Then combine with Eq.(15), the constrained system functions can be written as

$$
\left[\begin{array}{cc}
\boldsymbol{M} & \boldsymbol{C}_{\boldsymbol{Q}}^{\mathrm{T}} \\
\boldsymbol{C}_{\boldsymbol{Q}} & 0
\end{array}\right]\left[\begin{array}{l}
\ddot{\boldsymbol{Q}} \\
\boldsymbol{\lambda}_{c}
\end{array}\right]=\left[\begin{array}{c}
\boldsymbol{Q}_{v}-\boldsymbol{K} \boldsymbol{Q} \\
\tau
\end{array}\right]
$$

where $\tau=-C_{t t}-2 C_{Q t} \dot{Q}-\left(C_{Q} \dot{Q}\right)_{Q} \dot{Q}$.

Similar to the above steps substitute Eq.(19) and Eq.(17) into Eq.(26), we get the formula about $\left[\begin{array}{lll}q_{t}^{\mathbf{T}} & \lambda^{\mathbf{T}} & \lambda_{c}^{\mathbf{T}}\end{array}\right]_{n+1}^{\mathbf{T}}$

$$
\begin{aligned}
& {\left[\begin{array}{ccc}
\boldsymbol{M}_{t t} & \boldsymbol{M}_{t f} \boldsymbol{H}_{1} \mathrm{~d} t / 2 & \beta \mathrm{d} t^{2} \boldsymbol{C}_{1}^{\mathbf{T}} \\
\boldsymbol{M}_{t f}^{T} & \left(\boldsymbol{M}_{f f}+\beta \mathrm{d} t^{2} \boldsymbol{K}_{f f}\right) \boldsymbol{H}_{1} d t / 2 & \beta \mathrm{d} t^{2} \boldsymbol{C}_{2}^{\mathbf{T}} \\
\beta \mathrm{d} t^{2} \boldsymbol{C}_{1} & \beta \mathrm{d} t^{2} \boldsymbol{C}_{2} & 0
\end{array}\right]_{n+1}\left[\begin{array}{l}
\boldsymbol{q}_{t} \\
\boldsymbol{\lambda} \\
\boldsymbol{\lambda}_{\boldsymbol{c}}
\end{array}\right]_{n+1}} \\
& =\left[\begin{array}{l}
\left(\boldsymbol{Q}_{v \boldsymbol{n}+1}-\boldsymbol{M}_{\boldsymbol{n}+1} \ddot{\boldsymbol{Q}}_{n}^{\mathrm{int}}\right) \beta \mathrm{d} t^{2}-\boldsymbol{M}_{q} \\
\beta \mathrm{d} t^{2} \boldsymbol{\tau}_{n+1}
\end{array}\right]
\end{aligned}
$$

there

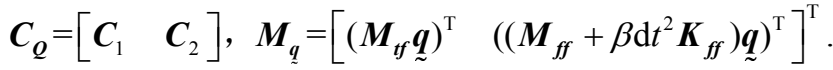

Employing Newton iterative strategy, the solution of Eq.(27) can be obtained.

\section{Numerical examples}

To validate the method proposed above, three numerical examples are presented in this section. The first example is a free-free rotating beam which illustrates the IBS method can be use in a flexible rotational system with one body. In this example the Lagrange interpolation function was used to reduce the elastic degrees. In the second example, a slider-crank system with two rotating beams validates this method for flexible multibody system.

\subsection{One free-free rotating beam}

The simple system is a free-free rotating beam. The whole structure is divided into 41 elements by finite element methods (FEM) and it contains 123 DOFs totally. The 
one-meter-long beam has a 5mm-radius circular cross section with Young's modulus 70Gpa and density $2.78 \mathrm{~g} / \mathrm{cm} 3$. It was excited by an impact force at the end, as shown in Figure. 2.

Y

$\mathbf{x}$ $\delta$

Figure. 2 One free-free rotating beam

If geometric nonlinearity is not considered in this example, the system can be described under linear FEM frame, thus

$$
\boldsymbol{M}^{e} \ddot{\boldsymbol{x}}+\boldsymbol{C}^{e} \dot{\boldsymbol{x}}+\boldsymbol{K}^{e} \boldsymbol{x}=\boldsymbol{F}^{e}
$$

where $\boldsymbol{M}^{e}, \boldsymbol{C}^{e}, \boldsymbol{K}^{e}$ are separately mass matrix, damper matrix, stiffness matrix and they are all constant matrix. $\boldsymbol{x}$ is element displacement vector and $\boldsymbol{F}^{e}$ is the external force vector. Using standard Newmark integration strategy shown in Eq.(19), it is easy to obtain the dynamic motion of this system. The motion of the beam during 0 to $0.8 \mathrm{~s}$ is shown in Figure. 3

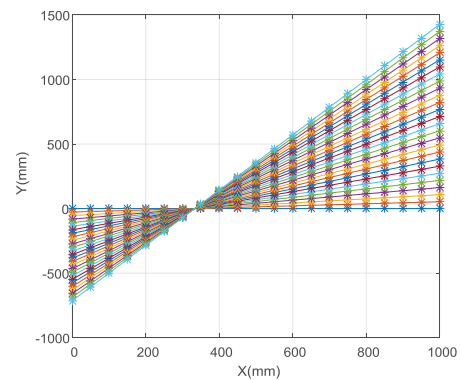

Figure. 3 The configuration of free beam's motion without consideration of geometric nonlinearity

Obviously, the result shown in Figure. 3 is not reasonable. Only displacement in $\mathrm{Y}$ direction is excited and the displacement in $\mathrm{X}$ direction is nearly zero. As a result, the beam seems to be stretched. The reason for this false phenomenon is the assumption of constant mass and stiffness matrix.

By introducing floating frame of reference and employing Eq.(20), we get a modified result and the motion of the beam during 0 to $0.8 \mathrm{~s}$ is illustrated in Figure. 4. This result is quite different from the above and is much more reasonable.

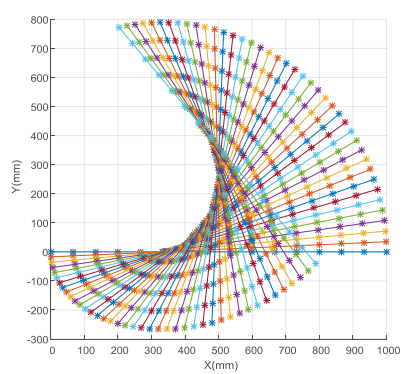

Figure. 4 The configuration of free beam's motion using floating frame of reference
To demonstrate the proposed method, in this example the solution of standard dynamic FE function under floating frame of reference is introduced as a benchmark which is described in Eq.(15).

To implement the IBS algorithm described in Eq.(20), the IRFs matrix $\boldsymbol{H}(t)$ should be obtained. As mentioned above, the motion of this beam can be divided into the moving of floating frame of reference (rigid motion) and local elastic vibration (flexible motion). In this example, the floating frame of reference is set at the position of one end of this beam and we assume that the structure is constrained by a pinned end and a fixed end shown in Figure. 5. Then the IRFs matrix of elastic vibration is achieved through this assumed constrained structure using FE method with Newmark integration strategy.

Figure. 5 Constrained structure with a pinned end and a fixed end

The external force applied to this system is

$$
F_{\text {ext }}= \begin{cases}1000 t & 0<t \leq 0.01 \\ 20-1000 t & 0.01<t \leq 0.02 \\ 0 & 0.02<t\end{cases}
$$

The velocity and acceleration of the end point in $\mathrm{Y}$ direction is presented in Figure. 6
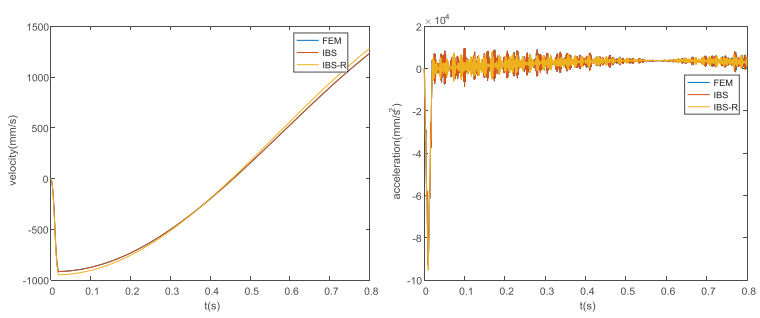

Figure. 6 Results between floating IBS, reduced floating IBS and traditional method

The results of floating IBS meet a great agreement with that of traditional method labeled as FEM in Figure. 6. With reduction of flexible DOFs, there are some errors produced between results of reduced floating IBS and traditional method. To present the accuracy quantitatively, $\mathrm{R}$ square(R2) and relative average absolute error(RAAE) are introduced and listed in Table 1. The large R2 and the smaller the RAAE, the more accurate results we obtain.

Table 1 Accuracy of floating IBS and reduced floating IBS compared with traditional method

\begin{tabular}{|c|c|c|c|r|}
\hline \multirow{2}{*}{} & \multicolumn{2}{|c|}{ floating IBS } & \multicolumn{2}{c|}{ reduced floating IBS } \\
\cline { 2 - 5 } & R2 & RAAE & R2 & RAAE \\
\hline V & 1.00000 & $3.09 \mathrm{e}-05$ & 0.99835 & 0.03564 \\
\hline A & 0.99842 & 0.02808 & 0.88014 & 0.24358 \\
\hline
\end{tabular}

The velocity and acceleration of the end point in frequency domain by Fourier transform is presented in 
Figure. 7. Over all, the results are acceptable and this method performs well at low frequency.
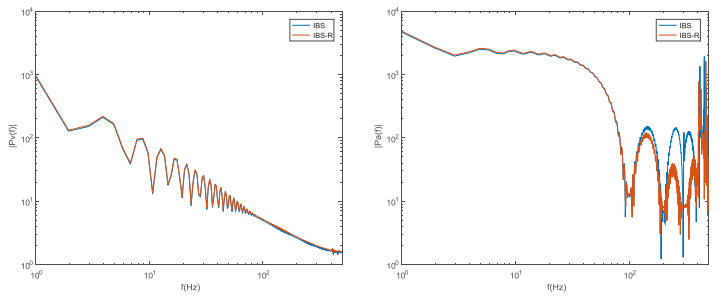

Figure. 7 Results in frequency domain between floating IBS and reduced floating IBS

\subsection{A slider-crank system with two rotating beams}

To validate the proposed method for multibody system, we introduce a slider-crank system. It consists of two beams and a mass block. They are connected by hinges and shown in Figure. 8.

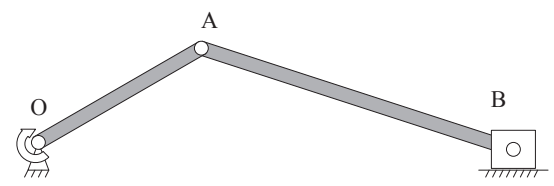

Figure. 8 A slider-crank system

In this system we assume beam OA has an initial angular velocity $25 \mathrm{rad} / \mathrm{s}$, mass block $\mathrm{B}$ has only one degree of freedom and they are connected by beam $\mathrm{AB}$ with hinges. The two beams have a $3 \mathrm{~mm}$-radius circular cross section with Young's modulus 200Gpa and density $7.87 \mathrm{~g} / \mathrm{cm} 3$. Beam OA and AB separately have a length of $0.15 \mathrm{~m}$ and $0.3 \mathrm{~m}$. The mass block $B$ has a same mass with beam OA. To make it simple, we ignore the flexibility of beam OA. The finite element model of beam AB is based on Euler-Bernoulli beam theory and the IRFs matrix used in Eq.(27) is obtained by this FE model. When calculating IRFs matrix, beam $\mathrm{AB}$ is assumed under the same constrained condition shown in Figure. 5. The system constrained conditions can be described as

$$
\left\{\begin{array}{l}
\boldsymbol{q}_{O \mathrm{X}}^{O A}=0, \quad \boldsymbol{q}_{O \mathrm{Y}}^{O A}=0 \\
\boldsymbol{q}_{A \mathrm{X}}^{O A}-\boldsymbol{q}_{A \mathrm{X}}^{A B}=0, \quad \boldsymbol{q}_{A \mathrm{Y}}^{O A}-\boldsymbol{q}_{A \mathrm{Y}}^{A B}=0 \\
\boldsymbol{q}_{B \mathrm{Y}}^{O A}=0
\end{array}\right.
$$

where $\boldsymbol{q}$ is the coordinate in the inertial reference, $\mathrm{X}$ and $\mathrm{Y}$ stand for the directions of $\boldsymbol{q}$, the superscript and subscript separately stand for the part and the point the coordinates belong to. Using generalized coordinates to express the Eq.(30), we get system constraint equations $\boldsymbol{C}$. Then the dynamic response is obtained by solving Eq.(27).

The angular displacement and angular velocity of beam OA are illustrated in Figure. 9. The results from floating IBS have a great agreement with that of traditional method. It demonstrates that this floating IBS is capable for multibody system with constraint equations.
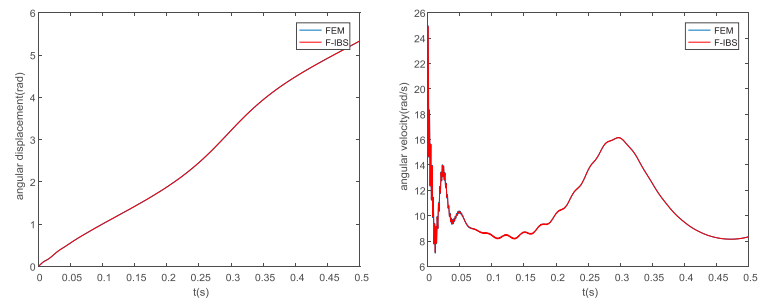

Figure. 9 Results of slider-crank system between floating IBS and traditional method

\section{Summary}

The proposed method extended the conventional IBS method. The introduction of the idea of floating frame of reference makes it possible to deal with the finite rotations of structures. The numerical examples demonstrate that this method can handle both unconstrained single-body system and constrained multibody system. With the employ of interpolation function, the local elastic DOFs are reduced. To some extent, this reduction of elastic DOFs will lead to some loss of simulating accuracy. In the mass, the results are acceptable and this method performs well at low frequency.

\section{References}

1. W. C. Hurty, Journal of the Engineering Mechanics Division 86 (4), 51-70 (1960).

2. B. Jetmundsen, R. L. Bielawa and W. G. Flannelly, Journal of the American Helicopter Society 33 (1), 55-64 (1988).

3. D. J. Rixen and P. L. C. van der Valk, Journal Of Sound And Vibration 332 (26), 7174-7190 (2013).

4. D. de Klerk, D. J. Rixen and S. N. Voormeeren, Aiaa Journal 46 (5), 1169-1181 (2008).

5. D. J. Rixen, Proceedings Of Isma2010 - International Conference on Noise And Vibration Engineering Including Usd2010, 1939-1954 (2010).

6. D. Rixen and N. Haghighat, presented at the 30th IMAC, Jacksonville, FL, United states, (2012).

7. M. V. van der Seijs and D. J. Rixen, Proceedings Of International Conference on Noise And Vibration Engineering (Isma2012) / International Conference on Uncertainty In Structural Dynamics (Usd2012), 3487-3499 (2012).

8. W.-L. Dong, L. Liu, S.-D. Zhou and S.-L. Chen, Aiaa Journal 53 (2), 504-509 (2015).

9. S.-D. Zhou, L. Liu and W.-L. Dong, AIAA Journal 54 (8), 2545-2551 (2016).

10. A. Shabana, Dynamics of multibody systems. (Cambridge University Press, 2005). 